\title{
Radial differential rotation vs. surface differential rotation: investigation based on dynamo models
}

\author{
Heidi Korhonen ${ }^{1}$ and Detlef Elstner ${ }^{2}$ \\ ${ }^{1}$ ESO, Karl-Schwarzschild-Str. 2, D-85748 Garching bei München, Germany \\ ${ }^{2}$ Astrophysical Institute Potsdam, An der Sternwarte 16, D-14482 Potsdam, Germany
}

\begin{abstract}
Differential rotation plays a crucial role in the alpha-omega dynamo, and thus also in creation of magnetic fields in stars with convective outer envelopes. Still, measuring the radial differential rotation on stars is impossible with the current techniques, and even the measurement of surface differential rotation is difficult. In this work we investigate the surface differential rotation obtained from dynamo models using similar techniques as are used on observations, and compare the results with the known radial differential rotation used when creating the dynamo model.
\end{abstract}

Keywords. Stars: rotation - stars: magnetic fields - stars: activity

\section{Model and analysis methods}

The model used in this work was originally used to investigate the flip-flop phenomenon (Elstner \& Korhonen 2005, Korhonen \& Elstner 2005). In the current work is is used to study the correlations between surface and radial differential rotation. The dynamo is modelled with a turbulent fluid in a spherical shell. The internal rotation is similar to the solar one with equatorial regions rotating faster than the polar regions, but here a smaller difference between core and surface rotation is used. The inner boundary of the convective zone is at the radius $0.4 \mathrm{R} \star$. The mean electromotive force contains an anisotropic alphaeffect and a turbulent diffusivity. The nonlinear feedback of the magnetic field acts on the turbulence only. The boundary conditions describe a perfect conducting fluid at the bottom of the convection zone and at the stellar surface the magnetic field matches the vacuum field.

We use standard cross-correlation methods to study the changes in the magnetic pressure maps obtained from the dynamo calculations. As these maps can be treated the same way as the temperature maps obtained from Doppler imaging, we can use the same techniques as in the case of the real observations to analyse the snapshots from the dynamo calculations. We have taken magnetic pressure maps from 36 time points over the activity cycle. The chosen time pointsare separated by about 50 days (Fig. 1).

\section{Results}

The results from cross-correlating the 36 maps are shown in Fig. 2. The plots give the shift in $\%$ day for each latitude on the 'northern' hemisphere. The field migration in the model is removed by normalising to the shift at the lowest latitude used in the investigation $\left(2^{\circ}\right)$. The last plot in Fig. 2 shows the average of the measurements from the cross-correlations, with standard deviation of the measurements as the error. The 

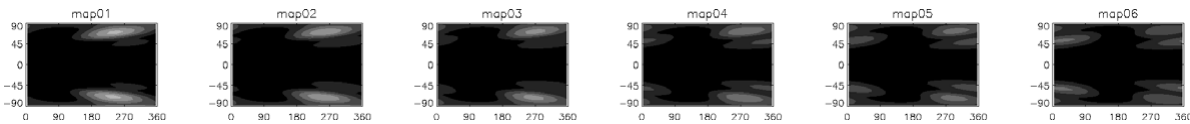

Figure 1. Six examples of the 36 snapshots of the Dynamo model.
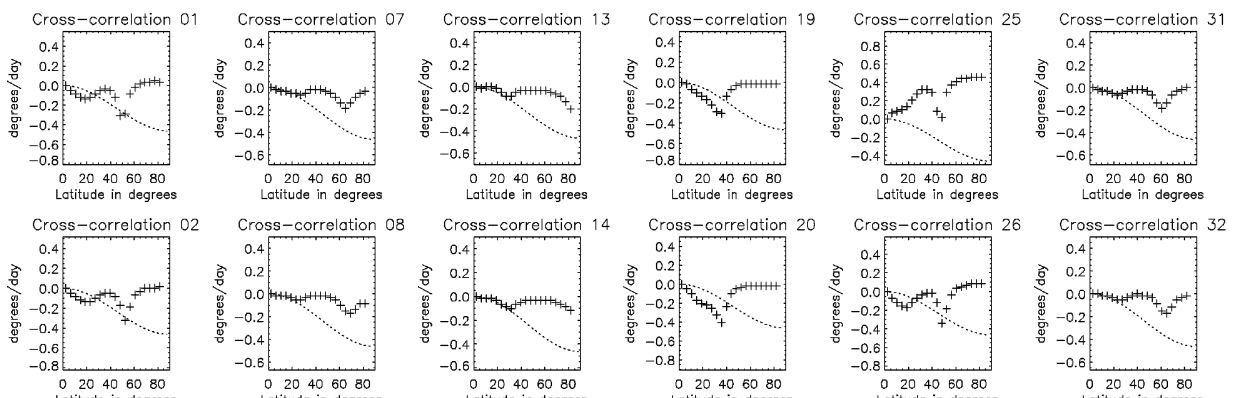

Cross-correlation 20
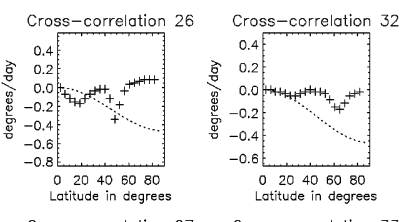

Cross-correlation 03
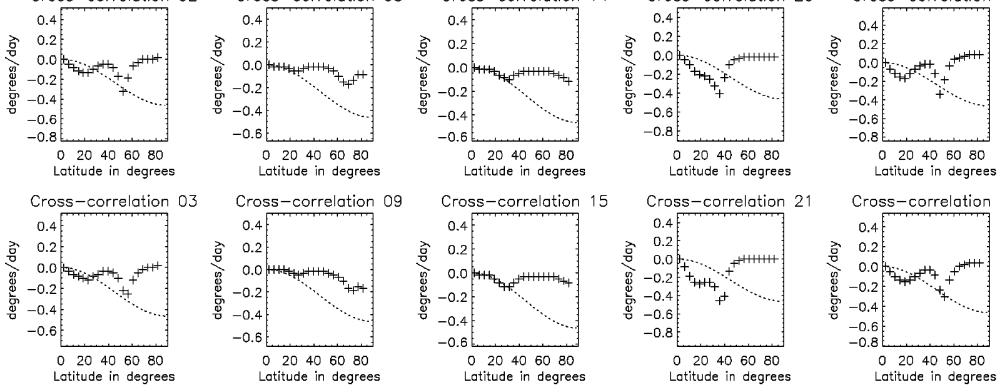

Cross-correlation 27

Cross-correlation 33
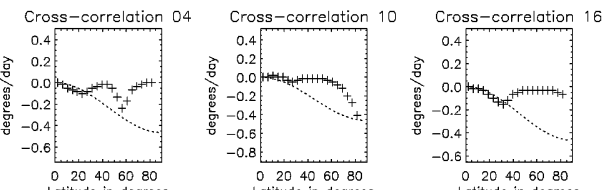

$0 \longdiv { 2 0 4 0 6 0 8 0 }$

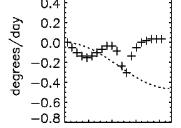

020406080
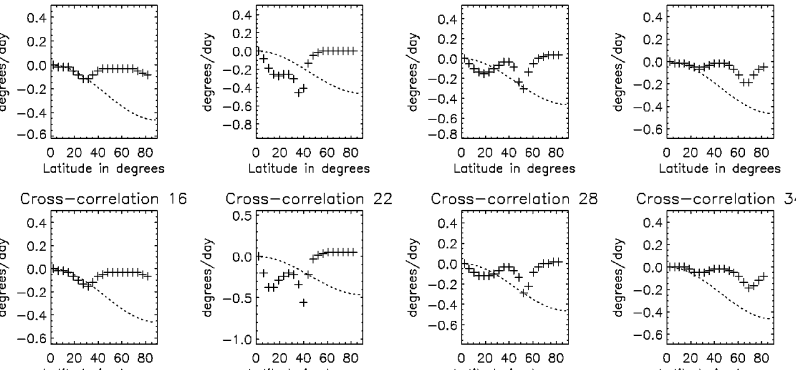

Cross-correlation 22

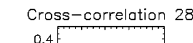

Cross-correlation 34
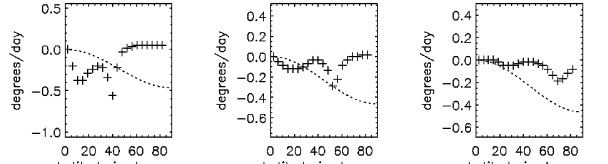

Cross-correlation 05

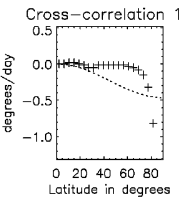

Cross-correlation 17
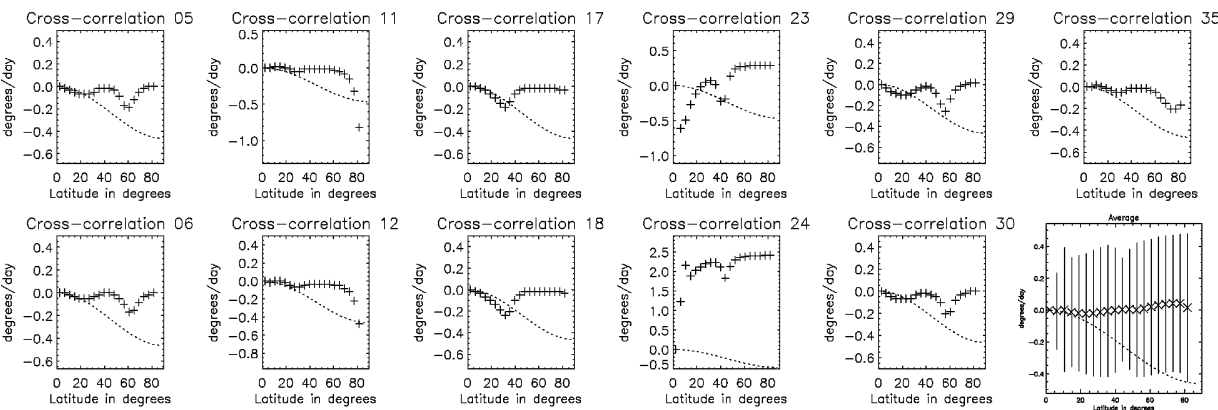

Figure 2. The results from cross-correlating the 36 snapshots.

dashed line is the input rotation at the stellar surface. The surface differential rotation pattern clearly changes during the activity cycle. It is also evident that the measured surface differential rotation at low latitudes is what one would expect from the model, but at higher latitudes, where most of the magnetic flux is, the correlation is very poor.

\section{Acknowledgements}

HK acknowledges the grant from IAU to help participating in the symposium.

\section{References}

Elstner, D. \& Korhonen, H. 2005, AN 326, 278

Korhonen, H. \& Elstner, D. 2005, A\&A 440, 1161 